\title{
Study on the Microbial Diversity of Rhizosphere Soil of Healthy Tobacco Plants and Plants Infected by Root-knot Nematode
}

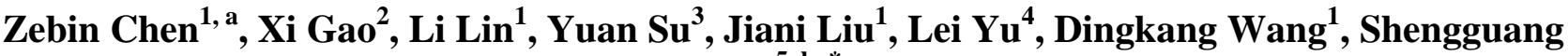 \\ $\mathrm{Xu}^{5, \mathbf{b}, *}$ \\ ${ }^{1}$ College of Agronomy, Kunming University, Kunming, Yunnan Province, China. \\ ${ }^{2}$ College of Plant Protection, Yunnan Agricultural University, Kunming, Yunnan Province, China. \\ ${ }^{3}$ Key Laboratory of Characteristic Biological Resource Development and Utilization of Colleges and \\ Universities in Yunnan Province, Kunming, Yunnan Province, China. \\ ${ }^{4}$ Yunnan Urban Characteristic Agriculture Engineering Technology Research Center, Kunming, Yunnan \\ Province, China. \\ ${ }^{5}$ Yunnan Institute of Biological Carbon Engineering Research Center, Kunming, Yunnan Province, China. \\ azbchenkmu@163.com, bsgxu2011@126.com \\ *Corresponding author: Shengguang Xu
}

Keywords: high throughput sequencing; root-knot nematode; tobacco; rhizosphere soil microorganism

\begin{abstract}
In order to understand the composition of microorganisms in rhizosphere soil of tobacco plants, the genome DNA of 16S rDNA-V4 region and ITS1 region of the rhizosphere soil of healthy tobacco plants and plants infected by root-knot nematode were sequenced through high throughput sequencing technique; Qiime and Mothur software were used to analyze the abundance and diversity of the flora. The results show that, the OTU abundance and $\alpha$ diversity index of bacteria and fungi in the soil of healthy plants were higher than those of the infected plants. Bacteroidetes group bacteria, which was dominant in farmland ecosystem, only accounted for $2.12 \%$ and $1.81 \%$ in the rhizosphere soil of healthy and infected tobacco plants. The abundance of Actinobacteria group, which has biocontrol effects, was higher in the rhizosphere soil of healthy plants than that of infected tobacco plants. It suggests that, the low abundance of Bacteroidetes and Actinobacteria groups may serve as an indicator of root-knot nematode infection. The analysis of community composition shows that, the abundance of Ascomycota groups in the rhizosphere soil of infected tobacco plants was significantly lower than that of the healthy plants; the abundance of Basidiomycota groups in the rhizosphere soil of infected plants was higher than that of the healthy plants. It could be inferred that the abundance of these two types of bacteria was related to the occurrence of root-knot nematode infection.
\end{abstract}

\section{Introduction}

Root-knot nematodes are common plant parasitic nematodes. In the whole world, the yield losses on most important cash crops caused by them are as high as tens of billions of dollars per year. [1] For many countries, the root-knot nematode infection is one of the main factors which limit the tobacco production. It causes increasingly serious harm to major tobacco fields in China. In Yunnan Province, twenty to thirty thousand hm2 tobacco are infected by root-knot nematode every year; disease strains cut down the tobacco production by 30\%-50\%. [2] At present, there are many related studies on the biological control of root-knot nematode. [3] But little research has been done into the ecological characteristics of soil microorganisms inhabited by root-knot nematode. Thus, substantial breakthroughs in the biological control of root-knot nematode cannot be made. Therefore, it is necessary to investigate the microbial diversity of rhizosphere soil of root-knot nematode infected tobacco plants and healthy tobacco plants, so as to fully understand the rules of root-knot nematode infection, and lay the foundation for its biological control. In the decades of research on soil microbial 
diversity, traditional isolation and culture techniques are used. But many studies have proved that microorganism identified by the traditional method only accounts for $0.1 \%-10 \%$ of the total environmental biomass, and cannot meet the requirements of microbial ecology study. [4] It is necessary for us to adopt non-culture techniques to study the real situation of biodiversity. In this study, the rhizosphere soil of root-knot nematode infected tobacco plants and healthy tobacco plants were used as experimental materials. The second generation high-throughput sequencing technique, which was developed in recent years, was used to analyze the soil microbial community structure in the rhizosphere soil. The aim of this paper was to provide theoretical basis for the pathogenesis and prevention of root-knot nematode disease.

\section{Research Materials and Research Method}

\subsection{Sample collection}

The soil samples were collected from tobacco field in Luoshui town of Xuanwei city. The soil was red soil and were commonly used to plant tobacco. The rhizosphere soil samples of root-knot nematode infected tobacco plants and healthy tobacco plants were collected and named as B1 and Z1 respectively. The surface soil was removed; the root soil was collected at the depth of $15-20 \mathrm{~cm}$ under the soil layer. The samples were immediately mixed and labeled after sieving by the $2 \mathrm{~mm}$ sieve, and then stored in the refrigerator at $4^{\circ} \mathrm{C}$.

\subsection{Total DNA extraction}

According to the instruction book of genomic DNA extraction kit produced by E.Z.N.A. Soil DNA Kit, the soil DNA was extracted. Then the purity and concentration of DNA were examined by 8g/L agarose gel electrophoresis. A proper amount of samples were taken to the centrifuge tube; the samples were diluted with aseptic water to $1 \mathrm{mg} \mathrm{L-1.}$

\subsection{PCR amplification}

The diluted genomic DNA was used as a template. To ensure the efficiency and accuracy of amplification, PCR was carried out by using high fidelity enzyme with specific primers of 16S-V4 zone and ITS1 region of Barcode (Phusion ${ }^{\circledR}$ High-Fidelity PCR Master Mix with GC Buffer produced by the New England Biolabs Company).

\subsection{Mixing and purification of PCR products}

The PCR product was detected by electrophoresis through $2 \%$ agarose gel. The target product was recovered by the gel extraction kit provided by Qiagen Company.

\subsection{Library construction and sequencing}

The library was built with library kit produced by TruSeq ${ }^{\circledR}$ DNA PCR-Free Sample Preparation Kit. The constructed library was quantified by Qubit and QPCR. After the library was qualified, the library was sequenced through MiSeq sequencer.

\subsection{Bioinformatics analysis}

First of all, the effective data (effective tags) was obtained after splicing original data with Flash software, filtering the data with Qiime software, and removing chimera with Uchime Algorithm software. Uparse software was used to cluster effective data on $97 \%$ level of the operational taxonomic unit (OTU). Greengene database was used in plant species annotation. Mothur software was used to do dilution curve analysis, and microbial community structure was obtained by analyzing the abundance and $\alpha$ diversity of OTU. 


\section{Results and Analysis}

\subsection{Analysis of OTU abundance and $\alpha$ diversity index of bacteria}

58268 original sequences were measured in the rhizosphere soil of infected plants (Table 1), and 54727 original sequences were measured in the rhizosphere soil of healthy plants. After filtering the low quality sequence, effective sequences in the rhizosphere soil of infected plants was 53972; 52545 effective sequences were obtained in the rhizosphere soil of healthy plants. At the similar level of 97\%, the bacterial sequence in the rhizosphere soil of infected plants could be divided into 1962 OTUs, and the bacterial sequence in the rhizosphere soil of healthy plants could be divided into 2204 OTUs. According to data about $\alpha$ diversity index, the Shannon index and Chao 1 index of bacteria in the rhizosphere soil of healthy plants were higher than those of the infected plants. The coverage rates of corresponding library were $96.36 \%$ and $95.81 \%$ respectively, indicating that the constructed library contained most bacterial groups, and could reflect the bacterial community structure of samples.

Table 1 OTU abundance and $\alpha$ diversity index of bacteria

\begin{tabular}{lllllll}
\hline \multirow{2}{*}{ sample } & $\begin{array}{l}\text { original } \\
\text { sequence } \\
\text { number }\end{array}$ & $\begin{array}{l}\text { Effective } \\
\text { sequence } \\
\text { number }\end{array}$ & $\begin{array}{l}\text { OUT } \\
\text { number }\end{array}$ & $\begin{array}{l}\text { shannon } \\
\text { index }\end{array}$ & $\begin{array}{l}\text { Chao1 } \\
\text { index }\end{array}$ & $\begin{array}{l}\text { Rate of } \\
\text { coverage }\end{array}$ \\
\hline B1 & 58268 & 53972 & 1962.0 & 9.162 & 2052.075 & 96.36 \\
Z2 & 54727 & 52545 & 2204.0 & 9.189 & 2332.278 & 95.81 \\
\hline
\end{tabular}

\subsection{Analysis of OTU abundance and $\alpha$ diversity index of fungi}

53892 original sequences were measured in the rhizosphere soil of infected plants (Table 2), and 36361 original sequences were measured in the rhizosphere soil of healthy plants. After filtering low quality sequence, the soil of infected plants had 52920 effective sequences; 35724 effective sequences were obtained from the soil of healthy plants. At the similarity level of $97 \%$, the sequence of bacteria in the rhizosphere soil of infected plants could be divided into 317 OTUs, and the sequence of bacteria in the soil of healthy plant could be divided into 350 OTUs. According to data about $\alpha$ diversity index, the Shannon index and Chaol index of fungi in the rhizosphere soil of healthy plants were higher than those of the infected plants. The coverage rates of corresponding library were $99.40 \%$ and $99.02 \%$ respectively, indicating that the constructed library contained most fungi groups, and could reflect the composition of fungi community in samples.

Table 2 OTU abundance and $\alpha$ diversity index of fungi

\begin{tabular}{|c|c|c|c|c|c|c|}
\hline \multirow[b]{2}{*}{ sample } & \multirow{2}{*}{$\begin{array}{l}\text { original } \\
\text { sequence } \\
\text { number }\end{array}$} & \multirow{2}{*}{$\begin{array}{l}\text { Effective } \\
\text { sequence } \\
\text { number }\end{array}$} & \multirow{2}{*}{$\begin{array}{l}\text { OUT } \\
\text { number }\end{array}$} & \multicolumn{3}{|c|}{$\alpha$ diversity index } \\
\hline & & & & $\begin{array}{l}\text { shannon } \\
\text { index }\end{array}$ & $\begin{array}{l}\text { Chao1 } \\
\text { index }\end{array}$ & $\begin{array}{l}\text { Rate of } \\
\text { coverage }\end{array}$ \\
\hline B1 & 53892 & 52920 & 317.0 & 4.709 & 330.220 & 99.40 \\
\hline Z2 & 36361 & 35724 & 350.0 & 5.001 & 377.364 & 99.02 \\
\hline
\end{tabular}

\subsection{Analysis on bacterial community composition}

Analysis from the perspective of phylum (Figure 1), bacteria in the rhizosphere soil of infected and healthy tobacco plants were mainly distributed in Proteobacteria (41.96\% and 27.49\%), Actinobacteria (19.28\% and 26.42\%), Acidobacteria (16.90\% and 16.70\%), Chloroflexi (5.39\% and 8.61\%), Gemmatimonadetes (5.42\% and 5.44\%), Crenarchaeota (1.22\% and 4.89\%), Planctomycetes (3.38\% and $4.34 \%)$, Bacteroidetes (2.12\% and $1.81 \%)$, Verrucomicrobia (1.12\% and $1.24 \%$ ), Nitrospirae (1.01\% and 0.94\%), and unknown groups (2.20\% and 2.11\%).

Analysis from the perspective of class (Figure 2), bacteria in the rhizosphere soil of infected and healthy tobacco plants were mainly distributed in Actinobacteria (10.21\% and 16.03\%), 
Alphaproteobacteria (14.22\% and 12.15\%), Acidobacteria-6 (13.65\% and 13.55\%), Gammaproteobacteria (13.20\% and 6.33\%), Betaproteobacteria (9.18\% and 4.94\%), Thermoleophilia (6.64\% and 6.72\%), Deltaproteobacteria (5.33\% and 4.02\%), Thaumarchaeota (1.22\% and 4.89\%), Ellin6529 (2.06\% and 4.40\%), Gemmatimonadetes (3.02\% and 2.79\%), and unknown groups (21.27\% and 24.19\%).

Analysis from the perspective of genus (Figure 3), bacteria in the rhizosphere soil of infected and healthy tobacco plants were mainly distributed in Candidatus Nitrososphaera (1.22\% and 4.83\%), Kaistobacter (3.69\% and 3.46\%), Marinobacter (1.84\% and $0.03 \%)$, Rhodoplanes $(1.01 \%$ and $0.85 \%)$, Pseudonocardia (0.66\% and $0.83 \%)$, Streptomyces ( $0.44 \%$ and $0.79 \%)$, Rubrobacter $(0.49 \%$ and $0.78 \%$ ), Methylophaga ( $0.77 \%$ and $0.02 \%)$, Hydrogenophaga (0.67\% and $0.04 \%)$, Planctomyces (0.37\% and $0.56 \%)$, and unknown groups (88.85\% and $87.80 \%)$.

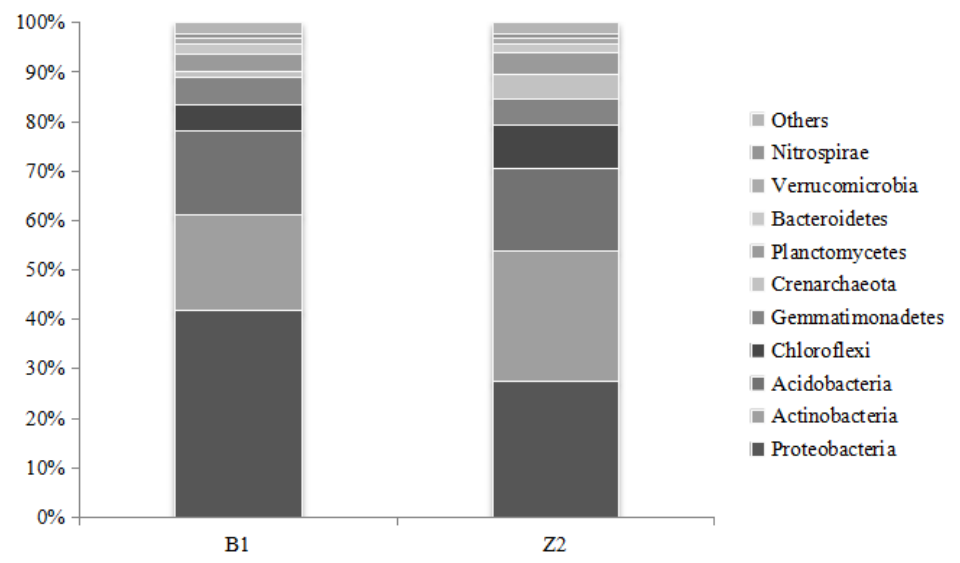

Figure 1. Bacterial Abundance and Composition from the Perspective of Phylum

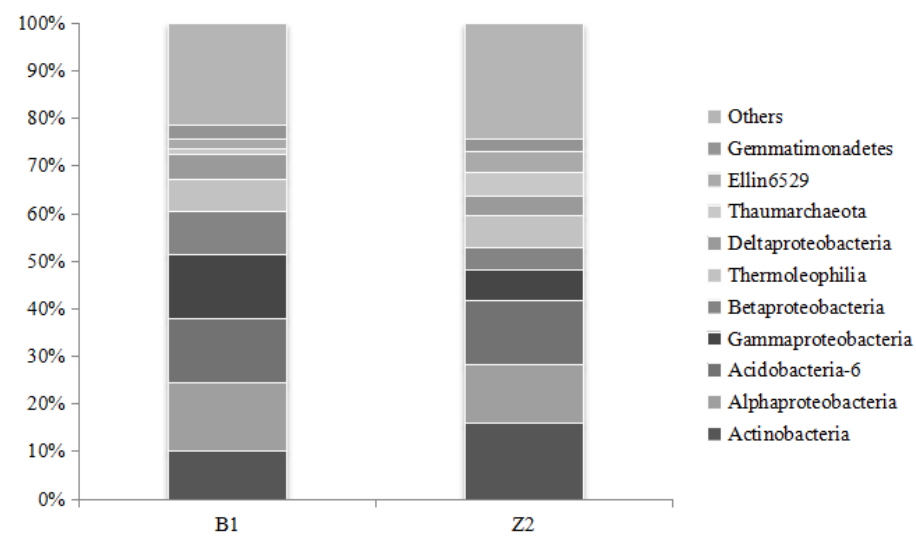

Figure 2. Bacterial Abundance and Composition from the Perspective of Class

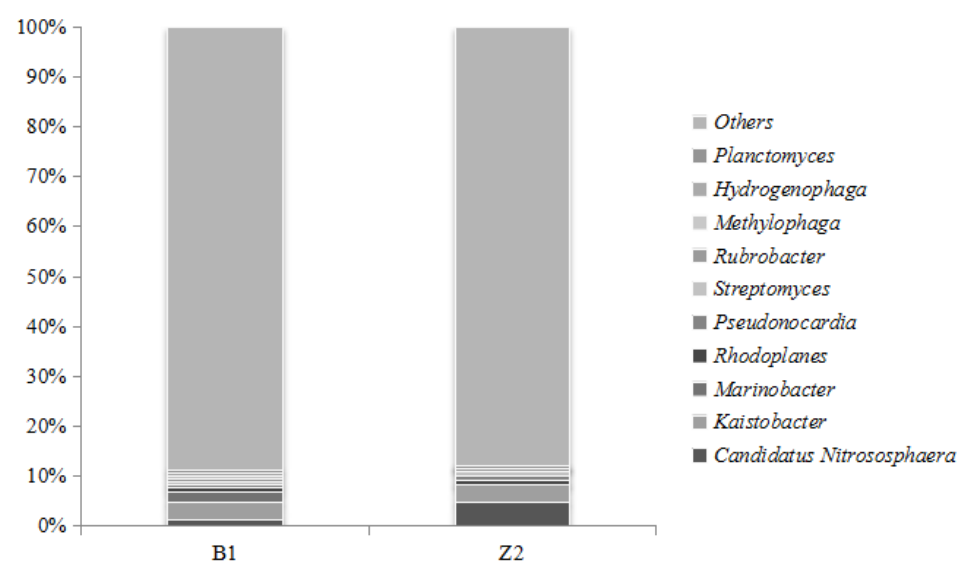

Figure 3. Bacterial Abundance and Composition from the Perspective of Genus 


\subsection{Analysis on fungi community composition}

Analysis from the perspective of phylum (Figure 4), fungi in the rhizosphere soil of infected and healthy tobacco plants were mainly distributed in Ascomycota (52.92\% and 80.82\%), Basidiomycota (8.50\% and 4.54\%), Zygomycota (3.12\% and 3.44\%), Un--s-Fungi sp (0.18\% and $0.46 \%$ ), Un--s-fungal sp ARIZ L453 (0.45\% and 0.34\%), Un--s-fungal endophyte $(0.44 \%$ and $0.42 \%)$, Un--s-fungal sp enrichment culture clone F2 (0.25\% and $0.43 \%)$, Un--s-fungal sp K6 (0.03\% and $0.15 \%)$, Un--s-fungal sp KRP24 (0.08\% and 0.07\%), Chytridiomycota $(0.01 \%$ and $0.04 \%)$, and unknown groups (34.01\% and 9.29\%).

Analysis from the perspective of class (Figure 5), fungi in the rhizosphere soil of infected and healthy tobacco plants were mainly distributed inSordariomycetes (29.22\% and 40.68\%), Eurotiomycetes (15.37\% and 27.60\%), Tremellomycetes (8.31\% and 3.91\%), IS--s-Retroconis sp XAE_090 (2.84\% and 7.21\%), IS--s-Mortierella sp VN2_2_5 (1.40\% and 1.70\%), Un--s-Ascomycota sp (1.58\% and 1.02\%), IS--s-Mortierella alpina (1.02\% and $1.34 \%)$, IS--s-Staphylotrichum sp $(0.96 \%$ and $1.18 \%)$, Dothideomycetes $(0.72 \%$ and $0.80 \%)$, Agaricomycetes (0.16\% and $0.62 \%$ ), and unknown groups (38.42\% and $13.94 \%$ ).

Analysis from the perspective of genus (Figure 6), fungi in the rhizosphere soil of infected and healthy tobacco plants were mainly distributed in Aspergillus (4.76\% and 14.79\%), Un--s-Sordariales sp $(4.32 \%$ and $12.46 \%)$, Corynascella $\quad(6.47 \%$ and $10.81 \%)$, Un--s-Tremellomycetes sp (8.28\% and 3.87\%), Chaetomium (8.20\% and 5.62\%), Retroconis (2.84\% and 7.21\%), Un--s-Chaetomiaceae sp (3.03\% and 6.43\%), Penicillium (4.62\% and 6.03\%), Chrysosporium (3.87\% and 5.41\%), Cylindrocarpon (2.45\% and $0.88 \%)$, and unknown groups (51.16\% and $26.49 \%)$.

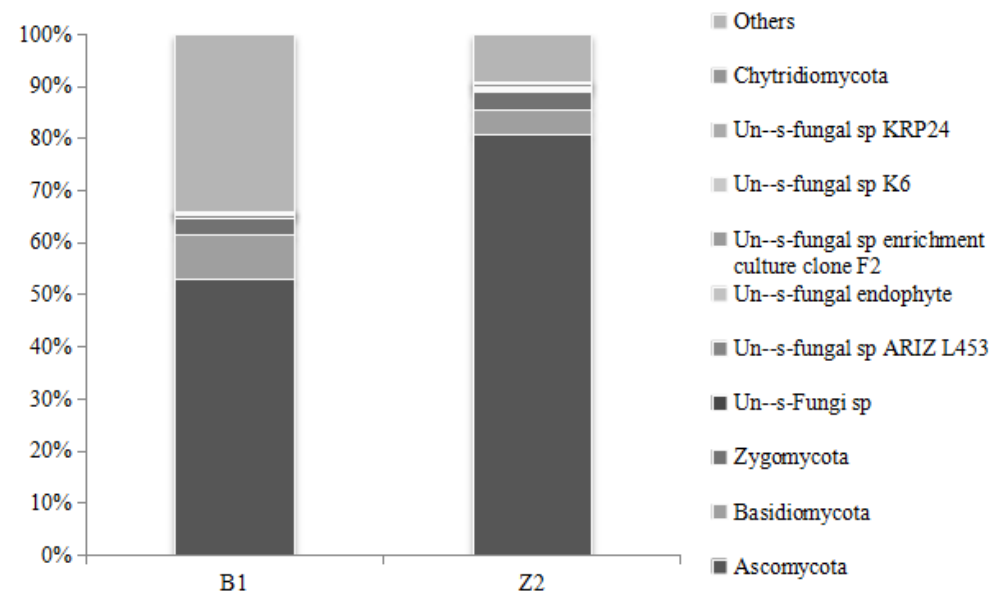

Figure 4. Fungi Abundance and Composition from the Perspective of Phylum

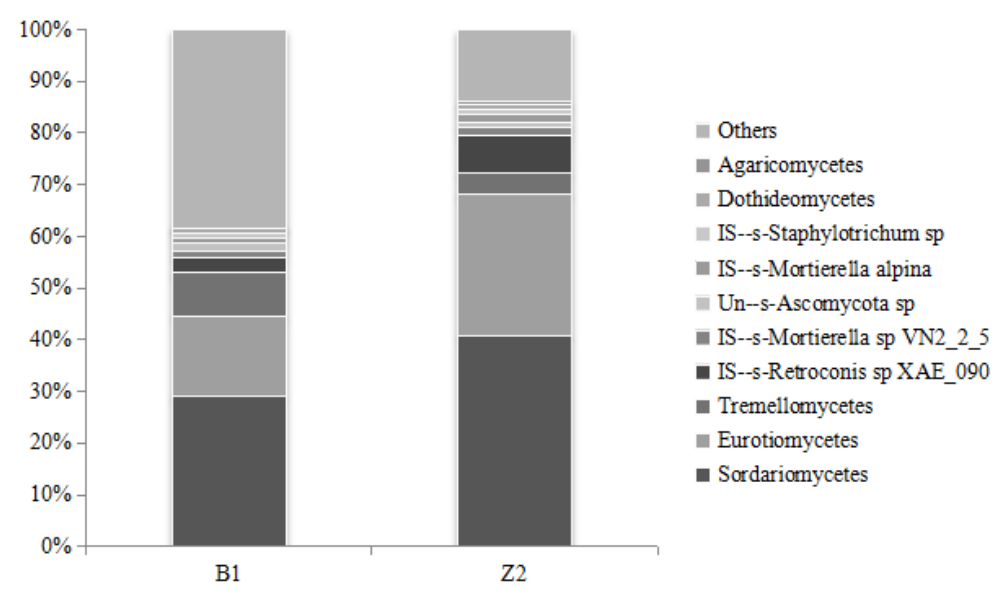

Figure 5. Fungi Abundance and Composition from the Perspective of Class 


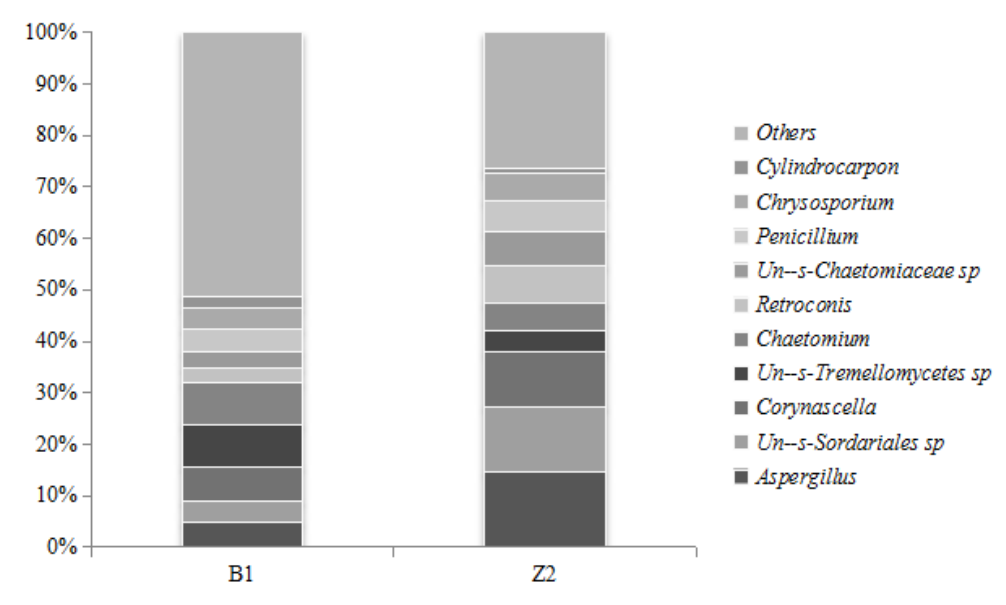

Figure 6. Fungi Abundance and Composition from the Perspective of Genus

\section{Conclusion and Discussion}

In this study, the rhizosphere soil samples of root-knot nematode infected tobacco plants and healthy tobacco plants were used as experimental materials. Illumina MiSeq high throughput sequencing technique was used to analyze the microbial community structure in the rhizosphere soil of tobacco plants. The defects of the first generation Sanger sequencing were overcome, while the microbial community structures were analyzed from the perspective of genome. The new method helps to break through the existing bottleneck, namely that many microorganisms still cannot be separated to cultivate. [5] Compared with the traditional isolation and culture method and non-culture method based on 16S rDNA analysis, high throughput sequencing technology covers almost all microbiological communities in the environment, and can detect low abundance microbiological species which could not be found in the past. [6]

None of the coverage of library reached $100 \%$, indicating that a few microbial communities were not detected. But this study only concerned the sequencing of DNA in 16S-V4 and ITS1 regions. More comprehensive results could be obtained in the future if other variable regions of DNA are sequenced.

The original sequence numbers of bacteria and fungi in the rhizosphere soil of infected plants were higher than those of healthy plants, indicating that numbers of bacteria and fungi in the rhizosphere soil of infected plants were higher than those of healthy plants. One possible reason is that, root-knot nematode causes the destruction of soil microbial ecosystem and changes the bacterial and fungal population structures in the rhizosphere soil; the number of beneficial microorganisms decreases while the number of pathogenic microbes increases, which leads to the enhancement of soil pathogenicity. [7] The OTU abundance and $\alpha$ diversity index were higher in rhizosphere soil of healthy plants than in rhizosphere soil of infected plants. Some studies showed, microbial diversity was related to the disease prevention effects of soil; the increase of soil microbial diversity could reduce the occurrence of soil-borne diseases. The greater of community diversity, the smaller of its dominance concentration will be; with more complex community structure, the community will be able to respond to environmental changes better and buffer its population fluctuation more effectively; strong feedback is the key to a stable community.[8] This study proved above opinion, and indicated that the key factor which determined the occurrence and severity of disease was the microbial structure in soil, rather than the number of microorganisms.

In current study about farmland soil bacterial diversity, Bacteroidetes group is considered as the dominant bacterial group regardless of crop species, soil physical and chemical properties, ecological factors and geographical locations. [9] But the results of this study showed that the proportions of Bacteroidetes group in the rhizosphere soil of infected and healthy tobacco plants were only $2.12 \%$ and $1.81 \%$. Compared with other cropland soil microbial systems, the abundance of this bacterial group is significantly unbalanced. The interaction between Bacteroidetes group and tobacco plants 
deserves further study. Actinobacteria group produces most of the antibiotics; the growth and reproduction of Actinobacteria group are important in adjusting the ecological balance of soil microbes. [10] In this study, though Actinobacteria group was one of the dominant groups in the rhizosphere soil of infected plants, its abundance in the rhizosphere soil was lower than that in the rhizosphere soil of healthy plants. It could be presumed that the decrease of its abundance was related to the occurrence of root-knot nematode. The low abundance of Bacteroidetes and Actinobacteria groups in soil might be regarded as the indicator of tobacco root-knot nematode disease.

The analysis of community composition showed that the dominant fungi in the rhizosphere soil of infected and healthy tobacco plants were Ascomycota (52.92\% and 80.82\%), Basidiomycota (8.50\% and $4.54 \%$ ) and Zygomycota (3.12\% and 3.44\%). In other soils, Ascomycota and Basidiomycota groups are also dominant fungi. Most of the fungi in these two types are soil inhabitants; they play an important role in maintaining the balance of soil microbial ecology by decomposing soil organic matter and plant root exudates.[11] This study found that, the abundance of Ascomycota groups in the rhizosphere soil of infected plants was significantly lower than that in the rhizosphere soil of healthy plants, while the abundance of Basidiomycota groups in the rhizosphere soil of infected plants was higher than that in the rhizosphere soil of healthy plants. It is inferred that the proportion of these bacteria groups was related to the root-knot nematode disease. Biological control fungi Chaetomium (8.20\% and 5.62\%) was also found in the rhizosphere soil of infected and healthy plants. The group contained a variety of biocontrol fungi, such as C. globosum which could inhibit a variety of soil borne pathogens; its antibacterial mechanism was to produce antibiotics and cell wall degradation enzyme. [12] C. globosum can reduce the incidence rate of rapeseed root swelling from $76.74 \%$ to 20\%. The C16 sphingosine and phytosphingosine produced by it can significantly reduce the germination rate of dormant Plasmodiophora brassicae spores. [13]

This study provides a more comprehensive analysis on the composition and diversity of microbes in the rhizosphere soil of infected and healthy tobacco plants. In the future, researchers can further study the relationship between root-knot nematode and the temporal and spatial variation of microbial populations, so as to select high effective biocontrol resources against root-knot nematode infection. By the combination of various biological control factors and the development of beneficial microorganism regulation methods, new clues could be found to effectively control the root-knot nematode disease in tobacco plantation.

\section{Acknowledgements}

This paper is supported by National Natural Science Foundation of China (41361056, 31460491); Joint Special Fundamental Research of Local Universities in Yunnan Province (2017FH001-041); Talent Introduction Program of Kunming University (YJL14005); Key Disciplines (Ecology) Project of Yunnan Education Department (05000511311); Open Fund Project of Key Laboratory of Characteristic Biological Resources Development and Utilization in Yunnan Province (GXKZ201716).

\section{References}

[1] P. Tan, R.G. Wang, G. Tian, et al. The influence of two kinds of tobacco root-knot nematode biocontrol bacteria on the number of microorganisms in soil, J. Hunan Agricultural Sciences. 4 (2016).

[2] J. Li, L.D. Zhang, F. Liu, et al. Effects of alkaline fertilizer on occurrence of banana wilt disease and soil microbial community, J. Journal of Plant Nutrition and Fertilizer. 22 (2016).

[3] R.P. Zhang, Q.B. Zeng, W. Yu, et al. Different measures of controlling tobacco toot-knot nematode disease in the field, J. Chinese Tobacco Science. 37 (2016).

[4] X.F. Li, Y.M. Wang, C.L. Xu, et al. Nematicidal activity of marigold against tobacco root-knot nematode, J. Journal of Anhui Agricultural Sciences. 45 (2017). 
[5] Y.Y. Ma, D.S. Wang, Y.L. Li, et al. Micro-ecology of the rhizoshpere soil of the phytophthora blight infected plants and healthy plants, J. Acta Agriculturae Boreali-occidentalis Sinica. 24 (2015).

[6] H.Y. Yu, G.Z. Lv, X.D. Sun, et al. Study on the species and quantity of rhizosphere soil fungi of healthy and root rot ginseng, J. Journal of Anhui Agricultural Sciences. 35 (2007).

[7] X.Y. Xu, Y.M. Yang, Y.M. Rui, et al. Preliminary identification of root-knot nematode species in Kunming flue-cured tobacco planting area, J. Journal of Yunnan Agricultural University (Natural Science). 32 (2017).

[8] L. Carpenterboggs, T.E. Loynachan, P.D. Stahl, Spore germination of Gigaspora margarita stimulated by volatiles of soil-isolated actinomycetes, J. Soil Biology \& Biochemistry. 27(1995).

[9] S.H. Zhang, Y.K. Wang, Q.G. Zhu, et al, Effect of adding wheat straw carbon sources to root-knot nematode diseased soil on soil microorganism community, J. Journal of Plant Nutrition and Fertilizer. 20 (2014).

[10] J.W. Huang, L.N. Tang, W.L. Zeng, et al, Effects of different soil treatments on biological characters of rhizosphere soil of flue-cured tobacco, J. Acta Tabacaria Sinica. 22 (2016).

[11] Y. Cao, N. Lu, X.J Chen, et al., Pyrosequencing analysis of soil bacteria composition in tobacco bacterial wilt disease nursery, J. Journal of Henan Agricultural Sciences. 46 (2017).

[12] M.Y. Gu, W.L. Xu, J. Mao, et al. Microbial community diversity of rhizosphere soil in continuous cotton cropping system in Xinjiang, J. Acta Ecologica Sinica. 32 (2012).

[13] W. Qiang, S. Guo, A.H. Zhang, et al. Screening antagonistic microorganisms against ginseng from Panax ginseng rhizosphere soil, J. Journal of South China Agricultural University. 35 (2014). 